\title{
Emergent rank-5 nematic order in $\mathrm{URu}_{2} \mathrm{Si}_{2}$
}

\author{
Hiroaki Ikeda ${ }^{1 \star}$, Michi-To Suzuki ${ }^{2}$, Ryotaro Arita ${ }^{3}$, Tetsuya Takimoto ${ }^{4}$, Takasada Shibauchi ${ }^{1}$ \\ and Yuji Matsuda ${ }^{1}$
}

\begin{abstract}
Exotic electronic states resulting from entangled spin and orbital degrees of freedom are hallmarks of strongly correlated $f$-electron systems. A spectacular example is the so-called hidden-order (HO) phase transition' ${ }^{1}$ in the heavy-electron metal $\mathrm{URu}_{2} \mathrm{Si}_{2}$, which is characterized by the huge amount of entropy lost at $T_{\mathrm{HO}}=17.5 \mathrm{~K}$ (refs 2,3 ). However, no evidence of magnetic/structural phase transition has been found below $T_{\text {HO }}$ so far. The origin of the HO phase transition has been a long-standing mystery in condensed-matter physics. Here, on the basis of a first-principles theoretical approach, we examine the complete set of multipole correlations allowed in this material. The results uncover that the $\mathrm{HO}$ parameter is a rank-5 multipole (dotriacontapole) order with nematic $E^{-}$symmetry, which exhibits staggered pseudospin moments along the [110] direction. This naturally provides comprehensive explanations of all key features in the $\mathrm{HO}$ phase including anisotropic magnetic excitations, the nearly degenerate antiferromagnetic-ordered state and spontaneous rotational-symmetry breaking.
\end{abstract}

In the rare-earth and actinide compounds, $f$ electrons behave like well-localized moments at high temperatures. As the temperature is lowered, $f$ electrons begin to delocalize owing to the hybridization with conduction electron wavefunctions. At yet lower temperatures the $f$ electrons become itinerant, forming a narrow conduction band with heavy effective electron mass, which is largely enhanced from the free-electron mass. Notable many-body effects within the narrow band lead to a plethora of fascinating physical phenomena including multipole order, quantum phase transition and unconventional superconductivity. Among them, perhaps the appearance of a $\mathrm{HO}$ state in $\mathrm{URu}_{2} \mathrm{Si}_{2}$ is one of the most mysterious phenomena. Identification of the microscopic order parameter and mechanism that drives the $\mathrm{HO}$ transition continues to be a central question in strongly correlated $f$-electron systems ${ }^{1}$.

There are several unique features that seem to be clues for understanding the $\mathrm{HO}$ in $\mathrm{URu}_{2} \mathrm{Si}_{2}$. In the paramagnetic state above $T_{\mathrm{HO}}$, the magnetic susceptibility exhibits the Ising-like anisotropy ${ }^{2,4}$. In the $\mathrm{HO}$ state below $T_{\mathrm{HO}}$, an electronic excitation gap is formed on a large portion of the Fermi surface $e^{5,6}$ (FS) and most of the carriers disappear ${ }^{7,8}$. Closely related to this, the gap formation also occurs in the magnetic excitation spectra at commensurate and incommensurate wave numbers, $\mathbf{Q}_{\mathrm{C}}=\left(\begin{array}{lll}0 & 0 & 1\end{array}\right)$ and $\mathbf{Q}_{\mathrm{IC}}=\left(\begin{array}{lll}0.6 & 0 & 0\end{array}\right)$, respectively, as revealed by the neutron inelastic scattering ${ }^{9-11}$. The HO ground state changes to the large-moment antiferromagnetic (AFM) state with the ordering vector $\mathbf{Q}_{\mathrm{C}}$ on applying hydrostatic pressure ${ }^{12,13}$, but the FS has a striking similarity between these different phases $^{14,15}$, implying that the HO is nearly degenerate with the AFM order. The magnetic torque measurements reveal the nematicity, which breaks the in-plane rotational (tetragonal) symmetry in the
$\mathrm{HO}^{16}$. The challenge for the theory has been to identify the order parameter that explains all of the above key features.

The theories that have been proposed to describe the HO state can be divided into two prevailing approaches; one is based on the localized $5 f$-electron model ${ }^{17-23}$ and the other the itinerant one $^{24-29}$. Recent angle-resolved photoemission spectroscopy results clearly demonstrate that all $5 f$ electrons are itinerant ${ }^{30}$ and the crystalline electric field, which is a signature of the localized nature, has never been observed. Moreover, the nuclear magnetic resonance measurements ${ }^{31}$ show a formation of the coherent heavy-electron state well above $T_{\mathrm{HO}}$. Therefore, it is natural to discuss the electronic structure on the basis of the itinerant picture. However, reliable calculation of the physical quantities by taking into account the complicated band structure is a difficult task. For this purpose, we use a state-of-the-art $a b$ initio downfolding (Supplementary Section SI) and dissect the electronic structure obtained from the density-functional theory (DFT) calculations. The obtained tight-binding Hamiltonian is constructed from 56 orbitals of $\mathrm{U} 5 f, \mathrm{U} 6 d, \mathrm{Ru} 4 d$ and $\mathrm{Si} 3 p$. Introducing the on-site Coulomb interactions between $5 f$ electrons, we obtain a realistic itinerant model, that is, a 56-band Anderson-lattice model including the spin-orbit interaction. On the basis of this realistic model Hamiltonian, magnetic and multipole correlations are analysed by the random-phase approximation (RPA) and beyond. To account for the mass renormalization effect in the Fermi-liquid theory, the energy and temperature scale is reduced by a factor of 10 throughout this study ${ }^{2,14,15}$, which makes comparisons to the experiments straightforward.

Figure 1 shows the paramagnetic FS and the band structure near the Fermi level, respectively. The energy bands crossing the Fermi level have mainly the total angular momentum $j=5 / 2$ multiplet of $U 5 f$. Each $j_{z}$ component of $j=5 / 2$ multiplet is coloured by weight. It turns out that each separated FS is mainly composed of a rather specific $j_{z}$ component without large mixing, except for the outer FS around the $Z$ point (Fig. 1). Such a $j_{z}$ component map is quite useful in that we are able to capture valuable information such as which parts of the FS play an essential role for the $\mathrm{HO}$ formation. Indeed, the disentanglement of FS orbital characters has also been an important theoretical advance to understand the electronic properties in iron-pnictide superconductors ${ }^{32}$.

First we discuss the RPA analysis of rank-1 (dipole) correlation, which is the conventional static magnetic correlation. The regime with $j_{z}= \pm 5 / 2$, shown in red, in the outer FS around the $\mathrm{Z}$ point is well nested with the outer FS around the $\Gamma$ point by the vector $\mathbf{Q}_{C}$, as indicated by the arrow in Fig. 1 (ref. 33). This nesting gives rise to a sharp peak of the correlation parallel to the $c$ axis (dipole $\left.J_{z}\right)$ at $\mathrm{Z}\left(\begin{array}{lll}0 & 0 & 1\end{array}\right)$ shown in Fig. 2a. Another salient feature is the hump structure at around $\left(\begin{array}{lll}0.6 & 0 & 0\end{array}\right)$ and the equivalent

${ }^{1}$ Department of Physics, Kyoto University, Kyoto 606-8502, Japan, ${ }^{2}$ CCSE, Japan Atomic Energy Agency, 5-1-5 Kashiwanoha, Kashiwa, Chiba 277-8587, Japan, ${ }^{3}$ Department of Applied Physics, University of Tokyo, Tokyo 113-8656, Japan, ${ }^{4}$ Asia Pacific Center for Theoretical Physics, POSTECH, Pohang 790-784, Korea. *e-mail: hiroaki@scphys.kyoto-u.ac.jp. 

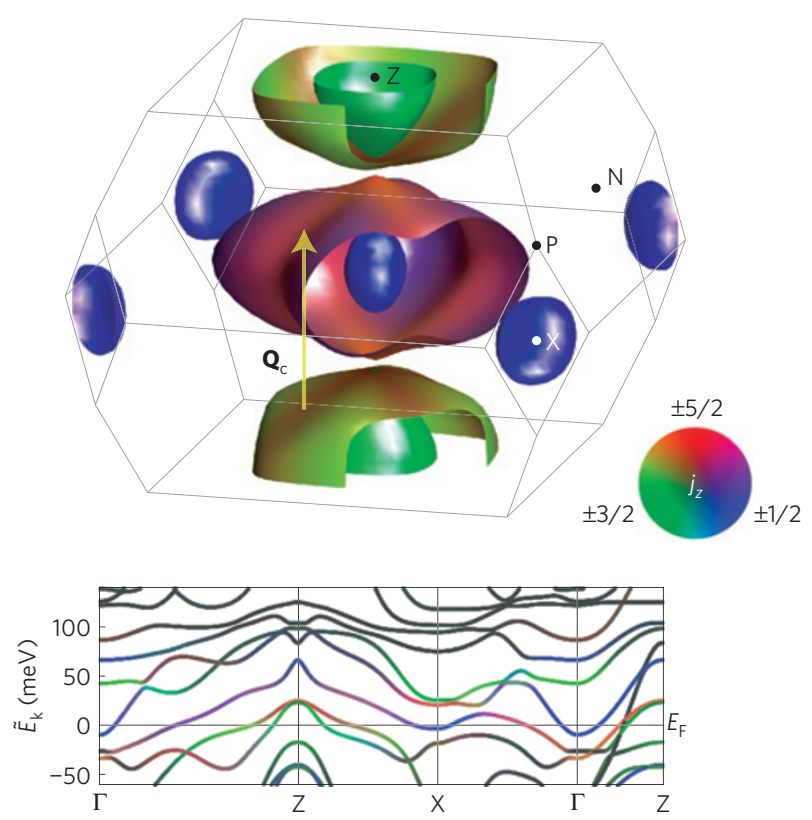

Figure 1 | Paramagnetic FS and energy band dispersion coloured by weight of the $j_{z}$ component. Red, green and blue colour gauges correspond to $j_{z}= \pm 5 / 2, \pm 3 / 2$ and $\pm 1 / 2$ components, respectively. The FS is constructed from two hole FSs around $Z$, and the other four electron FSs. Small (blue) electron pockets centred at $X$ and $\Gamma$ are constructed from the $j_{Z}= \pm 1 / 2$ component, and the inner (green) hole pocket around $Z$ is from $j_{Z}= \pm 3 / 2$. The outer hole pocket around $Z$ is a hybridized band between $\pm 3 / 2$ and $\pm 5 / 2$. The outer electron FS around $\Gamma$ is mainly composed of $j_{z}= \pm 5 / 2$, and partially hybridized with $\pm 1 / 2$. Two outer FSs around $\Gamma$ and $Z$ are partially nested with $\mathbf{Q}_{C}=(001)$ indicated by the arrow.

points, whose $\mathbf{Q}$-vectors coincide with $\mathbf{Q}_{\text {IC. }}$ We point out that these peak and hump structures in the paramagnetic phase are directly related to the magnetic excitation gap at $\mathbf{Q}_{C}$ and $\mathbf{Q}_{\text {IC }}$ in the HO phase ${ }^{11}$ because the gap opening occurs at the nested parts of the FS. We also note that the in-plane magnetic correlations, $J_{x}$ and $J_{y}$, are not enhanced in contrast to $J_{z}$ (Fig. 2b), which is also consistent with the Ising-like magnetic susceptibility ${ }^{2,4}$ and polarized-neutron measurements ${ }^{10}$.

Next we examine the higher-rank multipole correlations. According to the group theory, there are 36 multipole moments up to the fifth rank in $j=5 / 2$ subspace (Supplementary Table S1). Figure $2 \mathrm{c}-\mathrm{f}$ shows the correlations between the basis functions belonging to rank 2 (quadrupole), 3 (octupole), 4 (hexadecapole) and 5 (dotriacontapole), respectively. What is remarkable is that as in the case for the dipole $J_{z}$, the $\mathbf{Q}_{\mathrm{C}}$ correlation at the $\mathrm{Z}$ point is strongly enhanced in some cases such as $O_{20}(\operatorname{rank} 2), H_{x(y) b}(\operatorname{rank} 4), D_{4}$ (rank 5) and so on. Generally, these bases are mixed in the tetragonal symmetry, as shown by finite off-diagonal terms (red lines). The multipole correlations obtained by the diagonalization are depicted in Fig. 2g, in which each correlation at $\mathbf{Q}_{\mathrm{C}}$ is classified by the irreducible representations and the dominant component is denoted in parentheses (Supplementary Section SIII). At low temperatures, $A_{2}^{-}\left(J_{z}\right), E^{-}\left(D_{x(y)}\right)$ and $A_{1}^{-}\left(D_{4}\right)$ symmetries exhibit the first, second and third strongest enhancement. The first and the last two correspond to the AFM and dotriacontapole states, respectively. Within the RPA, the AFM state always overcomes the dotriacontapole states. To go beyond the RPA, we consider a staggered particle-hole pairing (generalized multipole orders at the $\mathrm{Z}$ point) mediated by the RPA fluctuation from an analogy with unconventional superconductivity (Supplementary Section SIV). This corresponds to the inclusion of the mode-mode coupling. From an analogue of the superconducting gap equation for the staggered ordering, we calculate the maximum eigenvalue $\lambda$. The corresponding multipole correlation increases proportionally to $\sim 1 /(1-\lambda)$, and then $\lambda=1$ provides the transition temperature. The temperature dependence of $\lambda$ of each symmetry is shown in Fig. 3a. As the temperature is lowered, $\lambda$ of $E^{-}\left(D_{x(y)}\right)$ is most strongly enhanced and the condition $\lambda=$ 1 is fulfilled at finite temperature, indicating a phase transition to the $E^{-}\left(D_{x(y)}\right)$ state. We emphasize that $E^{-}\left(D_{x(y)}\right)$ symmetry breaks the in-plane four-fold symmetry, which naturally accounts for the nematicity observed in the magnetic torque results ${ }^{16,34}$. In addition, the $E^{-}\left(D_{x(y)}\right)$ state breaks the time-reversal symmetry, which is consistent with the nuclear magnetic resonance measurements ${ }^{31}$. These lead us to conclude that the $\mathrm{HO}$ is $E^{-}\left(D_{x(y)}\right)$ dotriacontapole order.

The present calculations also reproduce well other key features of the $\mathrm{HO}$, that is, near degeneracy of the $\mathrm{HO}$ and AFM states and the anisotropic temperature dependence of the uniform susceptibility. Figure 3a demonstrates that $\lambda$ of $E^{-}\left(D_{x(y)}\right)$ is very close to that of $A_{2}^{-}\left(J_{z}\right)$. This indicates that both states are nearly degenerate and a small perturbation can change the HO to the AFM state. Indeed, we can construct a phase diagram by tuning the interactions (Supplementary Fig. S4), which is consistent with the pressuretemperature phase diagram (Fig. 3b). The temperature dependence of the uniform susceptibility $\chi_{c}(0)$ parallel to the $c$ axis exhibits a broad maximum at around $40 \mathrm{~K}$, whereas $\chi_{\mathrm{ab}}(0)$ perpendicular to the $c$ axis is smaller and nearly temperature independent (Fig. 3c), in good agreement with experiments ${ }^{2,4}$. The low-temperature decrease of $\chi_{c}(0)$ arises from the deep dip structure in the density of states near the Fermi level (Supplementary Fig. S1b). The Ising-like susceptibility including its temperature dependence has been discussed in terms of the crystalline electric field excitations of the localized $5 f$ electrons so far. However, the present results demonstrate that the susceptibility can be well accounted for by the itinerant scenario.

Why is such a high-rank multipole state (rank 5) with $E^{-}\left(D_{x(y)}\right)$ symmetry realized in $\mathrm{URu}_{2} \mathrm{Si}_{2}$ ? Similar high-rank multipole states have been proposed already; a rank- $4 A_{2}^{+}$state by the DFT+DMFT (dynamical mean field theory) method ${ }^{20}$, and a rank-5 $A_{2}^{-}$state by the DFT $+U$ method $^{27}$. However, these states obtained in the strong correlation limit are inconsistent with the nematic behaviour ${ }^{34}$. Detailed comparisons with other proposed HO parameters are listed in Supplementary Table S2. In our approach from the itinerant limit, the FS nesting with the $\mathbf{Q}_{C}$ vector plays an essential role in the multipole fluctuations. What is crucially important is that the FS regions connected by this $\mathbf{Q}_{\mathrm{C}}$ are dominated by the $\pm 5 / 2$ components, as shown in Fig. 1. In this situation, we can consider a subspace consisting of only two components $j_{z}=5 / 2$ and $-5 / 2$, which allows us to map $j_{z}= \pm 5 / 2$ to pseudospin $\uparrow$ and $\downarrow$. Then the dipole $J_{z}$ is described by the Pauli matrix $\sigma_{z}$ spanned in the pseudospin space, as it has only diagonal elements corresponding to $\pm 5 / 2 \Leftrightarrow \pm 5 / 2$. In the same way, $D_{x(y)}$ is given by $\sigma_{x(y)}$, representing off-diagonal components describing the $\pm 5 / 2 \Leftrightarrow \mp 5 / 2$ transition (Supplementary Section SV), which accompanies the angular momentum change of $5 \hbar$ allowed only in rank 5 . In this pseudospin space, the staggered $J_{z}$ state corresponds to the Néel order along the $c$ axis. On the other hand, the $D_{x(y)}$ state corresponds to the in-plane order breaking the rotational symmetry, where in-plane pseudospin moments are antiferromagnetically coupled along the $c$ axis (Fig. 3d). Thus, the pressure-induced first-order transition from the HO to AFM state can be explained by the pseudospin staggered moment flip from the in-plane to the out-of-plane direction. The experimentally observed nematicity along the [110] direction corresponds to the linear combination $D_{[110]}=(1 / \sqrt{2})\left(D_{x}+D_{y}\right)$ of the two-fold degenerate $D_{x}$ and $D_{y}$. The HO parameter is then represented by

$$
\phi_{[110]}(\mathbf{k})=\sum_{\alpha, \beta=\uparrow, \downarrow}\left\langle f_{\mathbf{k} \alpha}^{\dagger} \sigma_{[110]}^{\alpha \beta} f_{\mathbf{k}+\mathbf{Q}_{c} \beta}\right\rangle, \quad \sigma_{[110]}=\frac{\sigma_{x}+\sigma_{y}}{\sqrt{2}}
$$


a
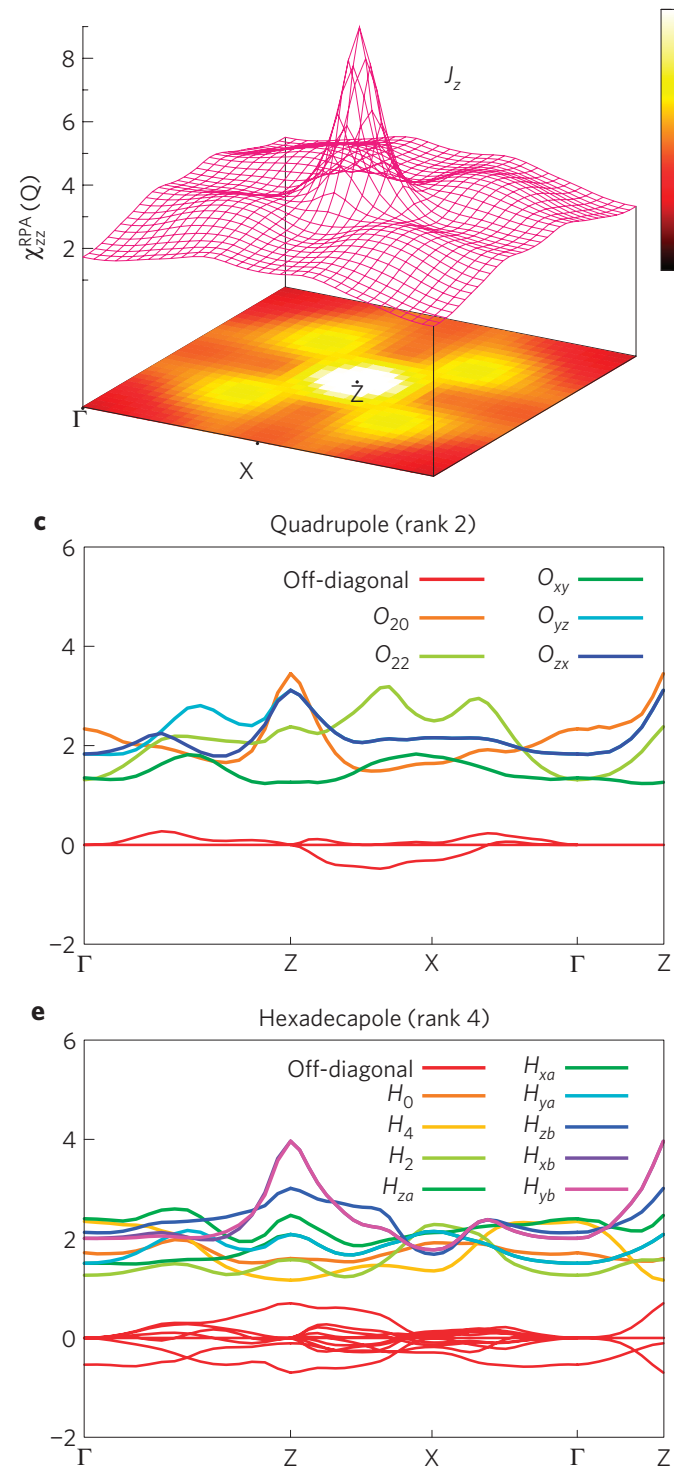

b

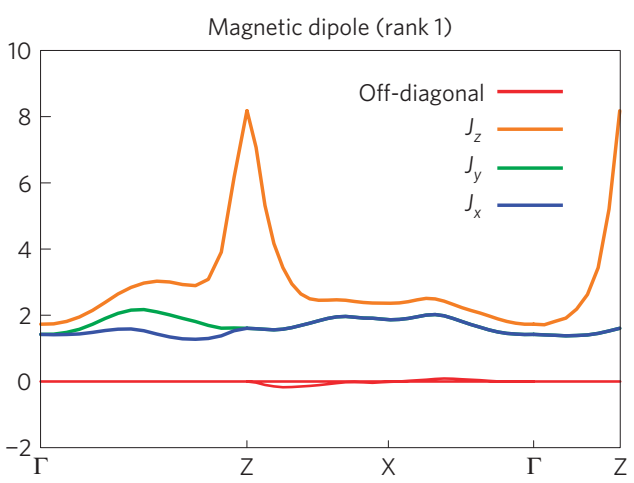

d

Octupole (rank 3)

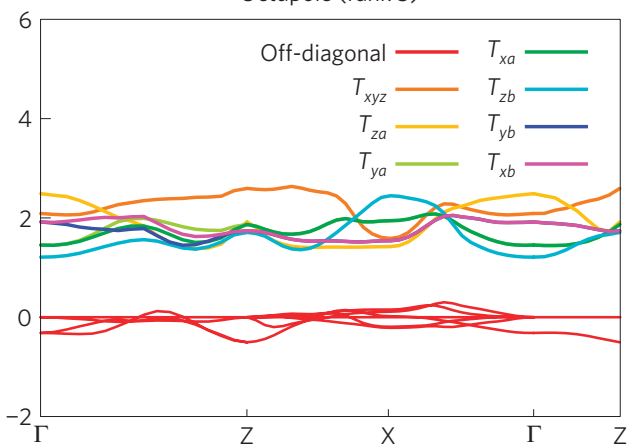

f

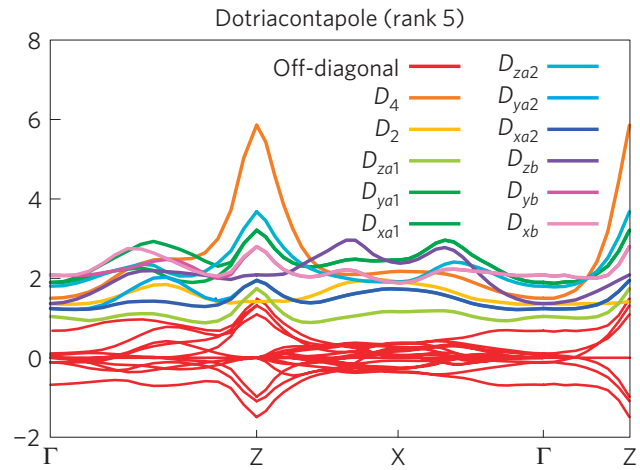

g

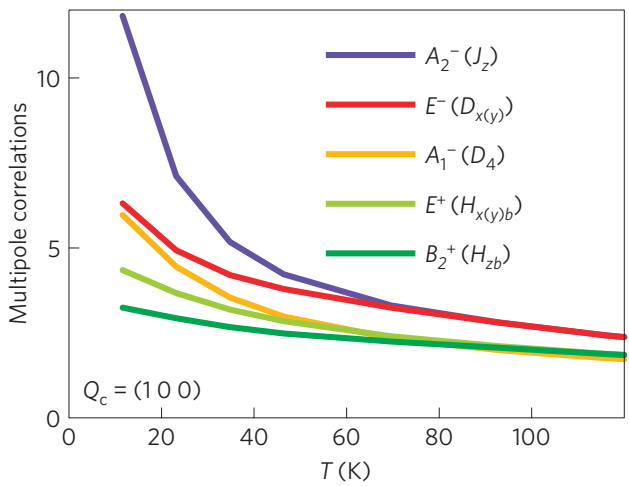

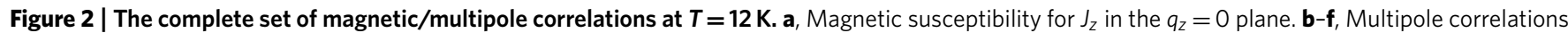
along the high-symmetry line for rank-1 dipole (b), rank-2 quadrupole (c), rank-3 octupole (d), rank-4 hexadecapole (e) and rank-5 dotriacontapole (f) basis functions. Off-diagonal correlations between different bases are also shown (red lines). g, Diagonalized multipole correlations as a function of temperature, where $D_{x(y)} \equiv\left(D_{x(y) a 1}+D_{x(y) a 2}+D_{x(y) b}\right) / \sqrt{3}$. All of these correlations have been obtained within the RPA calculations for $U=U^{\prime} \simeq 2.3$ and $J=J^{\prime}=0$ in units of $1 / \rho_{f}$, where $\rho_{f}$ is the total $f$-electron density of states at the Fermi level.

where $f_{\mathbf{k} \alpha}$ is an annihilation operator for an $f$ electron with momentum $\mathbf{k}$ and pseudospin $\alpha$. It should be noted that under in-plane $180^{\circ}$ rotation, the pseudospins change their direction, which discriminates this state from a nematic phase in the strict sense. However, its staggered nature leads to the two-fold nematic symmetry of the bulk susceptibility as observed experimentally.

Figure 4a shows the FS in the HO and AFM states, which is calculated by applying the effective multipole field so as to open the 
a

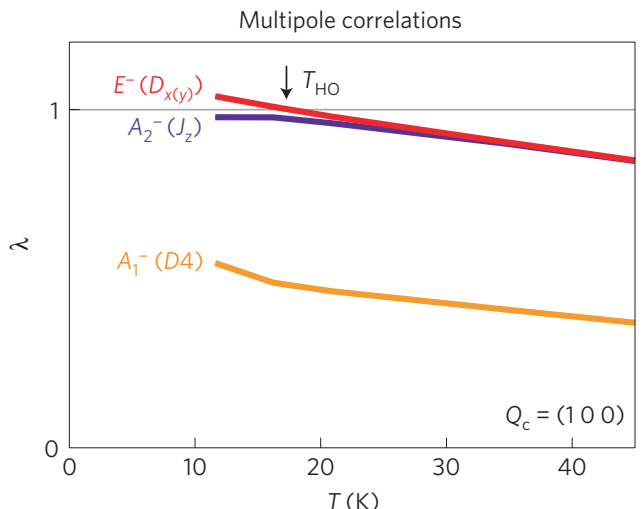

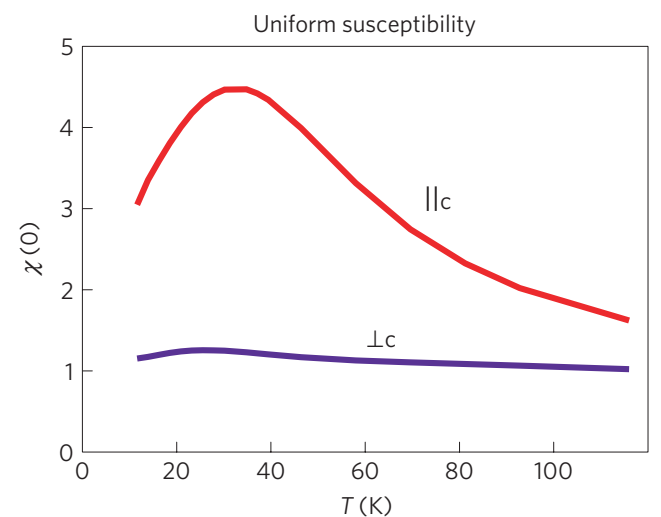

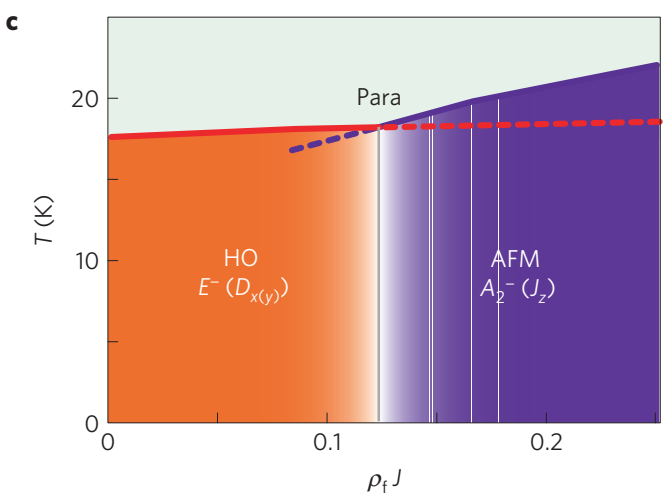

d
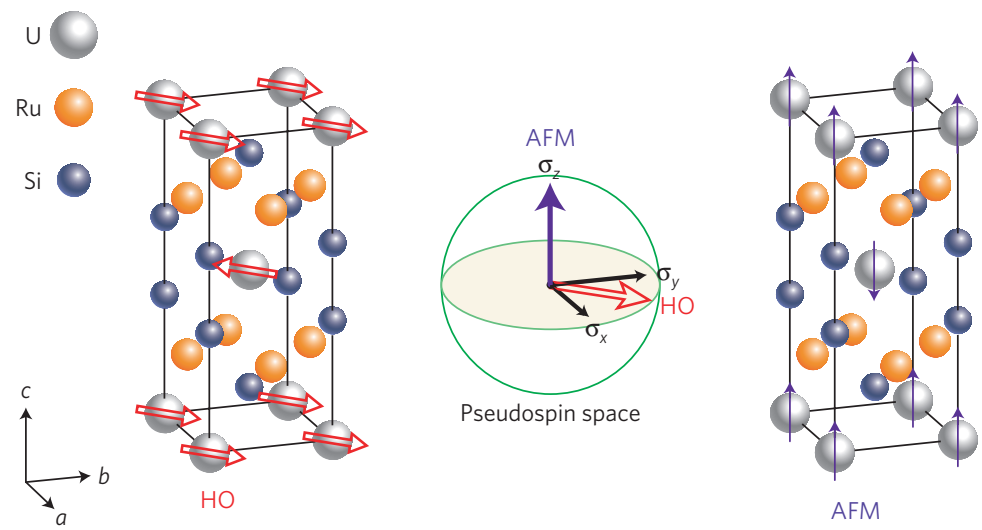

Figure 3 | Phase diagram and ordered structure. a, Temperature dependence of the maximum eigenvalue $\lambda$ in an analogue of the superconducting gap equation for the staggered ordering for $U=U^{\prime} \simeq 2.4$ and $J=J^{\prime}=0$. Note that $\lambda=1$ gives a phase transition temperature to the corresponding eigenstate. b. By increasing the Hund's coupling $J$ (Supplementary Fig. S4), the small difference in $\lambda$ between the rank-5 $E^{-}\left(D_{x(y)}\right)$ and rank-1 $A_{2}^{-}\left(J_{z}\right)$ states can be reversed, which may account for the pressure-induced AFM state. c, Temperature dependence of uniform susceptibilities parallel and perpendicular to the c axis calculated beyond the RPA. d, Schematic configurations of the $\pm 5 / 2$ pseudospin moments in the HO (left) and AFM (right) states are shown by the arrows. In both states, the pseudospins order antiferromagnetically along the $c$ axis, but the direction of the staggered moments in the pseudospin space differs between the two: along [110] for the HO state and along [001] for the AFM state (centre).

gap of $4 \mathrm{meV}$ observed by scanning tunnelling microscopy ${ }^{5,6}$. The lattice doubling in the AFM phase with $\mathbf{Q}_{\mathrm{C}}$ also occurs in the $\mathrm{HO}$ phase. Most of the FS having $j_{z}= \pm 5 / 2$ components disappears as a result of the gap opening at the nested parts of the paramagnetic FS. Around the $\Gamma$-point, small electron and large hole $(\alpha)$ pockets, the FS with a cage-like structure and four electron pockets $(\beta)$ exist. The FS in the HO phase bears a striking resemblance to that in the AFM state (Supplementary Section SVII), consistent with the quantum oscillation measurements. However, the broken four-fold symmetry in the HO state can be seen clearly in the FS with a cage-like structure (Fig. 4b), in sharp contrast to the AFM state.

The present approach based on the first-principles calculation is able to give a comprehensive explanation to the problem of $\mathrm{HO}$, which has been a quarter-century mystery. Why has the $\mathrm{HO}$ been hidden for a long time? The reason is that in conventional experimental techniques, such as resonant X-ray and neutron measurements, extremely highresolution measurements should be required for the direct detection of the high-rank multipole order parameter. We also point out that the present rank-5 order induces a very tiny but finite in-plane dipole moment belonging to the same symmetry $E^{-}$, which is roughly estimated as $10^{-2}-10^{-3} \mu_{\mathrm{B}}$ (Supplementary Section SVI). The detection of such a tiny moment remains a future issue. The itinerant multipole ordering with nematicity revealed in the present study is a new type of electron ordering, which is expected to be ubiquitously present in 
a

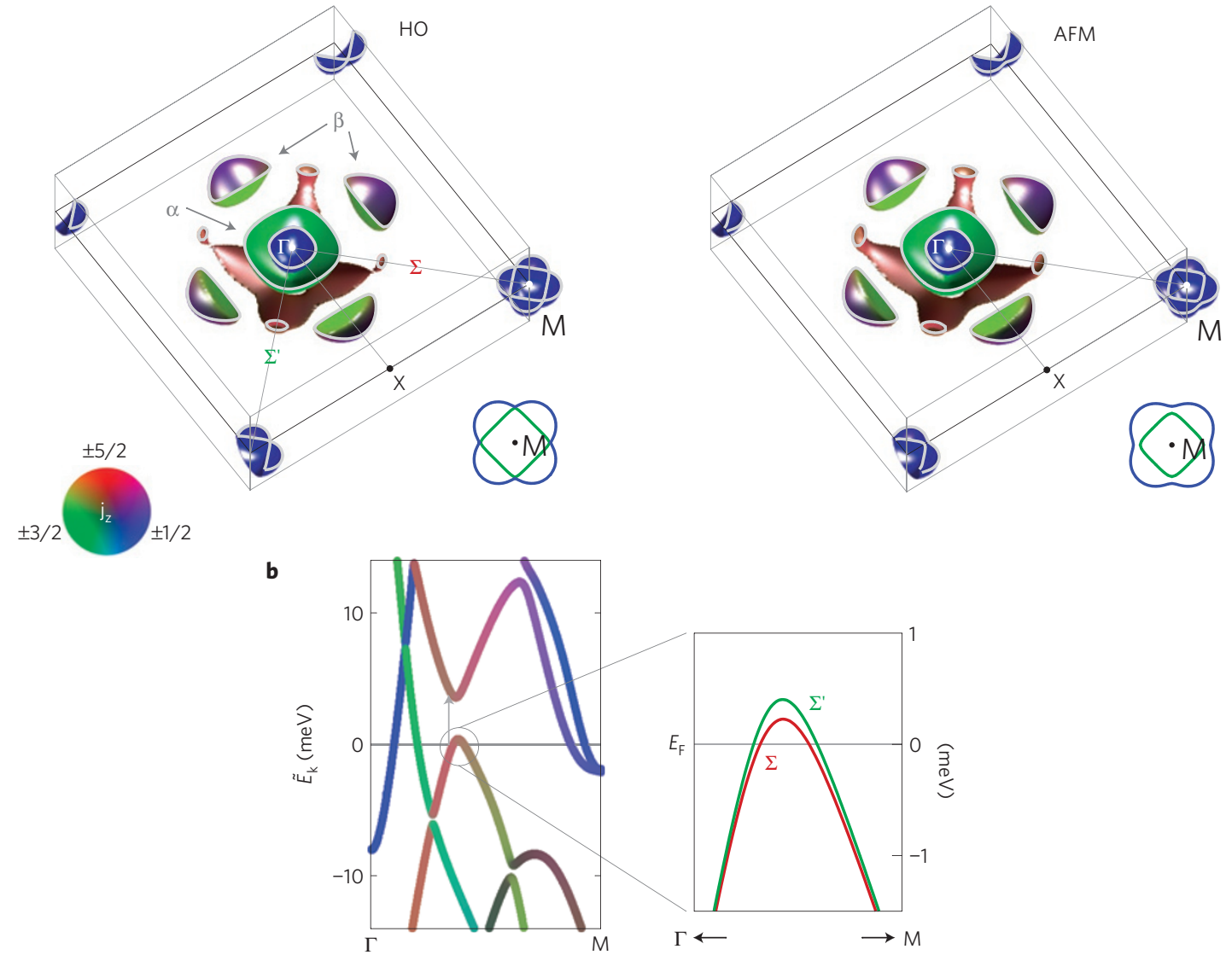

Figure $4 \mid \mathrm{HO}$ and AFM FSs. a, Two-dimensional cut of FSs at $k_{\mathrm{z}}=0$ in the $E^{-}\left(D_{[110]}\right)$ and $A_{2}^{-}$states. The Brillouin zone is folded with $\mathbf{Q}_{\mathrm{C}}=(001)$. The $D_{[110]}$ state (left panel) exhibits the in-plane four-fold symmetry breaking, which can be most easily seen in the cage FS. Two FSs around M (blue and green lines in the right bottom) in the $D_{[110]}$ state have almost no splitting along XM, which is in contrast to the large splitting found for the $A_{2}^{-}$AFM and the $E^{+}$ state (Supplementary Fig. S7). b, Band dispersion in the $D_{[110]}$ state along the $\Gamma \mathrm{M}$ line. The left panel highlights the excitation gap of $\sim 4$ meV (arrow). The magnification near the cage FS (right panel) shows a pronounced anisotropic $\Gamma M$ dispersion between $\Sigma$ (red) and $\Sigma^{\prime}$ (green) .

strongly correlated electron systems when spin and orbital degrees of freedom are entangled.

\section{Received 1 November 2011; accepted 27 April 2012;} published online 3 June 2012

\section{References}

1. Mydosh, J. A. \& Oppeneer, P. M. Colloquium: Hidden order, superconductivity and magnetism-the unsolved case of $\mathrm{URu}_{2} \mathrm{Si}_{2}$. Rev. Mod. Phys. 83, 1301-1322 (2011).

2. Palstra, T. T. M. et al. Superconducting and magnetic transitions in the heavy fermion system $\mathrm{URu}_{2} \mathrm{Si}_{2}$. Phys. Rev. Lett. 55, 2727-2730 (1985).

3. Maple, M. B. et al. Partially gapped Fermi surface in the heavy-electron superconductor $\mathrm{URu}_{2} \mathrm{Si}_{2}$. Phys. Rev. Lett. 56, 185-188 (1986).

4. Ramirez, A. P. et al. Nonlinear susceptibility as a probe of tensor spin order in $\mathrm{URu}_{2} \mathrm{Si}_{2}$. Phys. Rev. Lett. 68, 2680-2683 (1992).

5. Schmidt, A. R. et al. Imaging the Fano lattice to 'hidden order' transition in $\mathrm{URu}_{2} \mathrm{Si}_{2}$. Nature 465, 570-576 (2010).

6. Aynajian, P. et al. Visualizing the formation of the Kondo lattice and the hidden order in $\mathrm{URu}_{2} \mathrm{Si}_{2}$. Proc. Natl Acad. Sci. USA 107, 10383 (2010).

7. Behnia, K. et al. Thermal transport in the hidden-order state of $\mathrm{URu}_{2} \mathrm{Si}_{2}$. Phys. Rev. Lett. 94, 156405 (2005).

8. Kasahara, Y. et al. Exotic superconducting properties in the electron-hole-compensated heavy-Fermi semimetal $\mathrm{URu}_{2} \mathrm{Si}_{2}$. Phys. Rev. Lett. 99, 116402 (2007).

9. Broholm, C. et al. Magnetic excitations and ordering in the heavy-electron superconductor $\mathrm{URu}_{2} \mathrm{Si}_{2}$. Phys. Rev. Lett. 58, 1467-1470 (1987).

10. Bourdarot, F. et al. Precise study of the resonance at $Q_{0}=(1,0,0)$ in $\mathrm{URu}_{2} \mathrm{Si}_{2}$. J. Phys. Soc. Jpn 79, 064719 (2010).

11. Wiebe, C. R. et al. Gapped itinerant spin excitations account for missing entropy in the hidden-order state of $\mathrm{URu}_{2} \mathrm{Si}_{2}$. Nature Phys. 3, 96-99 (2007).

12. Amitsuka, H. et al. Pressure-temperature phase diagram of the heavy-electron superconductor $\mathrm{URu}_{2} \mathrm{Si}_{2}$. J. Magn. Magn. Mater. 310, 214-220 (2007)
13. Hassinger, E. et al. Temperature-pressure phase diagram of $\mathrm{URu}_{2} \mathrm{Si}_{2}$ from resistivity measurements and ac calorimetry: Hidden order and Fermi-surface nesting. Phys. Rev. B 77, 115117 (2008).

14. Ohkuni, H. et al. Fermi surface properties and de Haas-van Alphen oscillation in both the normal and superconducting mixed states of $\mathrm{URu}_{2} \mathrm{Si}_{2}$. Phil. Mag. B 79, 1045-1077 (1999).

15. Hassinger, E. et al. Similarity of the Fermi surface in the hidden order state and in the antiferromagnetic state of $\mathrm{URu}_{2} \mathrm{Si}_{2}$. Phys. Rev. Lett. 105, 216409 (2010).

16. Okazaki, R. et al. Rotational symmetry breaking in the hidden order phase of $\mathrm{URu}_{2} \mathrm{Si}_{2}$. Science 331, 439-442 (2011).

17. Santini, P. \& Amoretti, G. Crystal field model of the magnetic properties of $\mathrm{URu}_{2} \mathrm{Si}_{2}$. Phys. Rev. Lett. 73, 1027-1030 (1994).

18. Chandra, P. et al. Hidden orbital order in the heavy fermion metal $\mathrm{URu}_{2} \mathrm{Si}_{2}$. Nature 417, 831-834 (2002).

19. Kiss, A. \& Fazekas, P. Group theory and octupolar order in $\mathrm{URu}_{2} \mathrm{Si}_{2}$. Phys. Rev. B 71, 054415 (2005).

20. Haule, K. \& Kotliar, G. Arrested Kondo effect and hidden order in $\mathrm{URu}_{2} \mathrm{Si}_{2}$. Nature Phys. 5, 796-799 (2009).

21. Harima, H., Miyake, K. \& Flouquet, J. Why the hidden order in $\mathrm{URu}_{2} \mathrm{Si}_{2}$ is still hidden one simple answer. J. Phys. Soc. Jpn 79, 033705 (2010).

22. Kusunose, H. \& Harima, H. On the hidden order in $\mathrm{URu}_{2} \mathrm{Si}_{2}$ antiferro hexadecapole order and its consequences. J. Phys. Soc. Jpn 80, 084702 (2011).

23. Pépin, C. et al. A modulated spin liquid: A new paradigm for $\mathrm{URu}_{2} \mathrm{Si}_{2}$. Phys. Rev. Lett. 106, 106601 (2011).

24. Ikeda, H. \& Ohashi, Y. Theory of unconventional spin density wave: A possible mechanism of the micromagnetism in U-based heavy fermion compounds. Phys. Rev. Lett. 81, 3723-3726 (1998).

25. Varma, C. M. \& Zhu, L. Helicity order: Hidden order parameter in $\mathrm{URu}_{2} \mathrm{Si}_{2}$. Phys. Rev. Lett. 96, 036405 (2006).

26. Elgazzar, S. et al. Hidden-order in $\mathrm{URu}_{2} \mathrm{Si}_{2}$ originates from Fermi surface gapping induced by dynamic symmetry breaking. Nature Mater. 8 , 337-341 (2009). 
27. Cricchio, F., Bultmark, F., Granäs, O. \& Nordström, L. Itinerant magnetic multipole moments of rank five as the hidden order in $\mathrm{URu}_{2} \mathrm{Si}_{2}$. Phys. Rev. Lett. 103, 107202 (2009).

28. Dubi, Y. \& Balatsky, A.V. Hybridization wave as the hidden order in $\mathrm{URu}_{2} \mathrm{Si}_{2}$. Phys. Rev. Lett. 106, 086401 (2011).

29. Fujimoto, S. Spin nematic state as a candidate of the hidden order phase of $\mathrm{URu}_{2} \mathrm{Si}_{2}$. Phys. Rev. Lett. 106, 196407 (2011).

30. Kawasaki, I. et al. Band structure and Fermi surface of $\mathrm{URu}_{2} \mathrm{Si}_{2}$ studied by soft X-ray angle-resolved photoemission spectroscopy. Phys. Rev. B 83, 235121 (2011).

31. Takagi, S. et al. No evidence for small-moment antiferromagnetism under ambient pressure in $\mathrm{URu}_{2} \mathrm{Si}_{2}$ : Single-crystal ${ }^{29} \mathrm{Si}$ NMR study. J. Phys. Soc. Jpn 76, 033708 (2007).

32. Kuroki, K. et al. Unconventional pairing originating from the disconnected Fermi surfaces of superconducting $\mathrm{LaFeAsO}_{1-x} \mathrm{~F}_{x}$. Phys. Rev. Lett. 101, 087004 (2008).

33. Oppeneer, P. M. et al. Electronic structure theory of the hidden-order material $\mathrm{URu}_{2} \mathrm{Si}_{2}$. Phys. Rev. B 82, 205103 (2010).

34. Thalmeier, P. \& Takimoto, T. Signatures of hidden-order symmetry in torque oscillations, elastic constant anomalies, and field-induced moments in $\mathrm{URu}_{2} \mathrm{Si}_{2}$. Phys. Rev. B 83, 165110 (2011).

\section{Acknowledgements}

We thank K. Ueda, K. Haule, G. Kotliar, M. Sigrist and T. M. Rice for helpful discussions and suggestions. This work was supported by a Grant-in-Aid for the Global COE programme 'The Next Generation of Physics, Spun from Universality and Emergence', a Grant-in-Aid for Scientific Research on Innovative Areas 'Heavy Electrons' (20102002, 20102006) from MEXT, and KAKENHI from JSPS.

\section{Author contributions}

H.I. and R.A. developed a methodology for the DFT+RPA and beyond. M-T.S. analysed the fermiology in some ordered states. T.T. provided group-theoretical arguments of multipoles. H.I., T.S. and Y.M. wrote the text. All authors contributed to critical discussion of the physical interpretation of the results.

\section{Additional information}

The authors declare no competing financial interests. Supplementary information accompanies this paper on www.nature.com/naturephysics. Reprints and permissions information is available online at www.nature.com/reprints. Correspondence and requests for materials should be addressed to H.I. 